\title{
Effects of Packaging on Mechanical Stress in 3D-ICs
}

\author{
V. Cherman, M. Lofrano, V. Simons, M. Gonzalez, G. Van der Plas, J. De Vos, T. Wang, R. Daily, A. Salahouelhadj, \\ G. Beyer, A. La Manna, I. De Wolf*, E. Beyne. \\ IMEC \\ Kapeldreef 75, 3001, Leuven, Belgium \\ *also at Dept. of Materials engineering KU Leuven, Leuven, Belgium \\ vladimir.cherman@imec.be
}

\begin{abstract}
In this work the mechanical stress induced in 3D stacks by different packaging process steps is studied. The 3D stacks used in this work are assembled using two identical dies containing a number of stress sensors which are designed and manufactured in $65 \mathrm{~nm}$ technology. It is observed that the contribution of the package substrate and the die-attach process to the redistribution of mechanical stress inside the 3D stacked IC is more significant than the one of the EMC and that the influence of packaging on the shape and amplitude of local stress around the inter-die interconnects (micro-bumps) is not significant. These observations are supported by the measurements of stress done using micro-Raman spectroscopy and are correlated with the results of finite element modeling and with optical warpage measurements of different packaging configurations.
\end{abstract}

\section{Introduction}

3D integrated circuits (3D-IC) attract growing interest as they offer the unique opportunity of increasing the density of transistors per square centimeter of the package without the need of following the scaling Moor's law to miniaturize the latter. In addition, in comparison with hybrid integrated circuits, 3D-IC technology exhibits the shortest inter-chip interconnects thus significantly improving the power consumption, signal integrity and bandwidth [1]. It also offers virtually unlimited potential in combining different technologies and functionalities within one 3D-IC which, inturn, can be used for significant reduction of weight, volume and cost of the future microelectronic components and systems [2]. The key elements of the 3D-IC are vertical interconnects which combine different components and provide means of communication between chips within the integrated circuit. These components are the through silicon vias (TSVs) inside the chip(s) and the inter-die interconnects. The latter can be established by micro-solder joints or metalmetal direct bonds $[3,4]$. To improve the mechanical stability, an underfill (UF) material is used to glue the individual chips within the 3D stacked IC.

Despite of many advantages of the 3D-stacking approach, the addition of electrical and mechanical interfaces in combination with high-temperature processing steps can deteriorate the performance and the reliability of the individual ICs within the 3D stack [5]. The effects of the presence of $\mathrm{TSV}(\mathrm{s})$ on the FEOL devices [6,7] and on the performance of the BEOL interconnects [8] have been constantly addressed in the last decade. Moreover, the influence of stacking (inter-die interconnects and UF) has recently been investigated via the analysis of the particular test case where a special chip designed and manufactured in $65 \mathrm{~nm}$ technology was used $[9,10]$. This test chip contains n- and p-type field effect transistors (FETs) which are used as mechanical stress sensors due to their significant piezoresistive response. After calibration these sensors allow quantitative analysis of mechanical stresses induced in FEOL. In [9] it was shown that the difference in the coefficient of thermal expansion (CTE) between UF and microbump results in high vertical stress which in turn affects the electrical performance of the FEOL devices and may deteriorate the overall reliability of the $3 \mathrm{D}$ assembly.

In this work the 3D stacked test chips introduced in [9] have further been assembled in fcLFBGA packages, using different electronics mold compounds (EMCs). The aim of this study is to investigate the influence of packaging on the stress in the 3D-ICs.

\section{Packaging options}

The package configuration used in this work is flip-chip Low-Profile Fine-Pitch Bold Grid Array (fcLFBGA) with 676 IOs arranged in an array of 26 columns and 26 rows with $0.5 \mathrm{~mm}$ pitch. The cross section of the package is schematically shown in Figure 1.

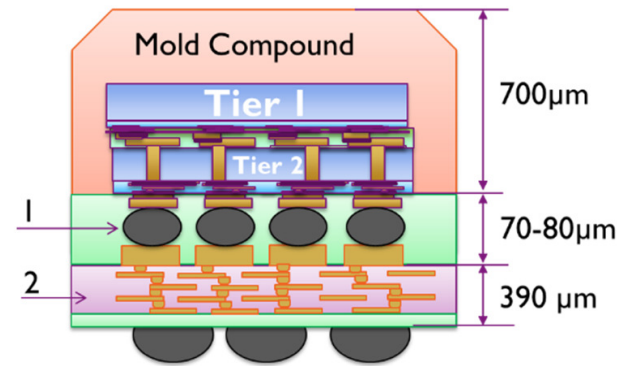

Figure 1. Schematic cross section of the packaged 3D stack. " 1 " indicates vertical interconnects consisting of $5 \mu \mathrm{m}$ thick, $50 \mu \mathrm{m}$ in diameter $\mathrm{Cu}$ pads, reflowed laser jetted micro-solder balls and the CUF, and " 2 " is the laminate substrate.

The package consists of the 3D stack described in details in [9], the package substrate ("2" in Figure 1) and the mold compound. It contains two dies, tier 1 (top die) and tier 2 (bottom die). The assembly sequence for the 3D package can be divided onto the following steps:

1. Jetting the micro-solder balls onto the $5 \mu \mathrm{m}$ thick, $50 \mu \mathrm{m}$ in diameter copper pads,

2. Flip-chip attachment of the 3D stack to the substrate with the following mass reflow cycles with the temperature budget of $\mathrm{T}=260^{\circ} \mathrm{C}$,

3. Applying capillary underfill (CUF) and its curing at $\mathrm{T}=150^{\circ} \mathrm{C}$ for 2 hours. 
4. Application the molding compound followed by its curing at $\mathrm{T}=180^{\circ} \mathrm{C}$,

5. BGA solder balls are attached to the pads on the back side of the substrate with the following reflow cycle.

The processing steps 1-3 are indicated as "1" in Figure 1.

The packages with the electronics mold compound (EMC) are used to investigate the effect of the EMC on the mechanical stress induced in the 3D-IC. This type of package can also be used to experimentally investigate the thermal behavior of the 3D stack inside the package under the low power operation regime [11].

Another packaging option is achieved by skipping step \#4 ("Application the molding compound and its curing") from the above list. In this case the final package does not have the protective mold compound and the backside of the 3D stack as well as the parts of the laminate substrate and the undefilled interfaces (die-die interface with no-flow underfill (NUF) and stack-substrate with CUF) are exposed to the environment. This type of package is used to separate the effects of different packaging steps (i.e. die-attach and overmolding) on the mechanical stress induced in the 3D stacks. In terms of thermal behavior, the package with the exposed (bare) die can be used in combination with the heat sink (or other external cooling solution) attached to the back side of the stack. Thus, the thermal regimes at very high power dissipation can be experimentally investigated [11].

In this work all packages use a $390 \mu \mathrm{m}$ thick 4-layer laminate substrate (" 2 " in Figure 1) where the Young's modulus and the coefficient of thermal expansion of the core component are $28 \mathrm{GPa}$ and $20 \mathrm{ppm} /{ }^{\circ} \mathrm{C}$, respectively. The $3 \mathrm{D}$ stack described in detail in [9] is assembled to the substrate by the flip-chip die-attach process. Images of the $14 \mathrm{~mm} \times 14 \mathrm{~mm}$ packages with and without the mold compound are shown together with the image taken from the side of the BGA balls in Figure 2.
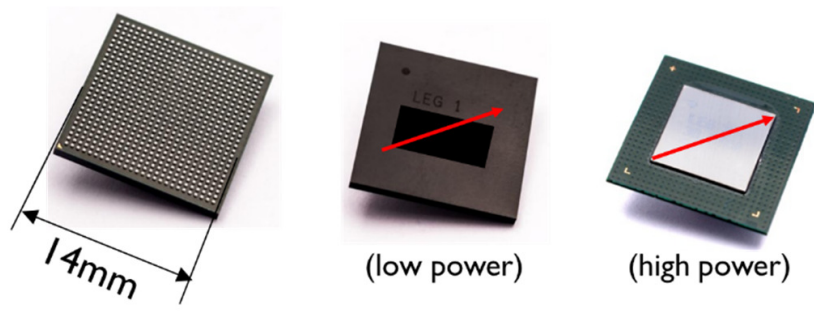

Figure 2. 3D-stacked IC packages. From left to right: view from the BGA balls side, top view of the overmolded package and top view of the bare-die package. Arrows indicate the diagonal on which sensors reported in the following paragraphs are located.

The three packaging options are listed in Table 1 which contains the information about the mechanical and thermomechanical properties of two different EMCs. The last column in the table indicates the number of packaged samples contributing to the statistical analysis of the results.
Table 1. Packaging options

\begin{tabular}{|c|c|c|c|c|}
\hline $\begin{array}{c}\text { EMC } \\
\text { type }\end{array}$ & $\begin{array}{c}\mathbf{C T E} \\
{\left[\mathbf{p p m} /{ }^{\circ} \mathbf{C}\right]}\end{array}$ & $\begin{array}{c}\mathbf{E} \\
{[\mathbf{G P a}]}\end{array}$ & $\begin{array}{c}\mathbf{T g} \\
{\left[{ }^{\circ} \mathbf{C}\right]}\end{array}$ & $\begin{array}{c}\text { Sample } \\
\text { Size }\end{array}$ \\
\hline EMC2 & 10 & 29 & 120 & 29 \\
\hline EMC4 & 12 & 20 & 175 & 18 \\
\hline NoEMC & - & - & - & 15 \\
\hline
\end{tabular}

The first two options represent the packages with different mold compounds. These mold compounds have similar coefficients of thermo-mechanical (CTE) but are about 50\% different in terms of the glass transition temperature of the mold, $T_{g}\left(120^{\circ} \mathrm{C}\right.$ vs. $\left.175^{\circ} \mathrm{C}\right)$ and in terms of Young's modulus of the mold at temperatures below $T_{g}, E$ (29GPa vs. 20GPa).

The electrical access to the package' IOs is established through the dedicated printed circuit board (PCB) and the socket compatible with the packages (as shown in Figure 3). The drawback of using such a socket for accurate measurements of mechanical stress induced by packaging is the sample mounting. To establish a reliable mechanical and electrical connection between the BGA balls and the socket contacts, the package is pushed vertically against the board using a screw integrated into the top part of the socket. Thus additional vertical stress is induced into the package from the measurement setup. This stress is expected to be negligibly small and is not considered in this work.

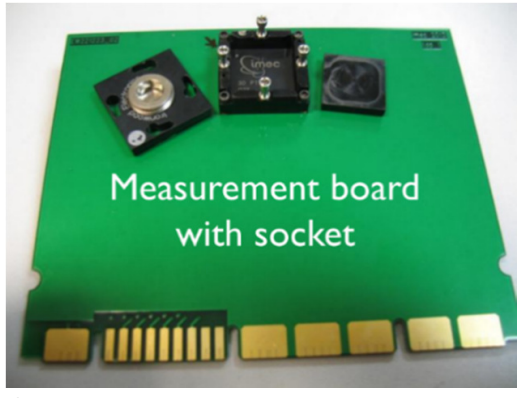

Figure 3. Measurement board with the socket.

\section{Mechanical stress induced by packaging}

To analyze the effects of packaging on the mechanical stress the set of stress sensors ( $\mathrm{n}$ - and p-type FETs) is measured on every package. This is the same set of sensors which was used to investigate the effects of 3D stacking in [9]. The deviations of the saturation currents of the sensors from their initial values measured at the stack level (before packaging) are used as the measure of the stress induced by the packaging processes. The relative changes of the saturation currents $\left(\Delta \mathrm{I}_{\mathrm{ON}}\right)$ of global stress sensors as the result of packaging are shown in Figure 4 for the nine pre-defined locations along the diagonal of the $3 \mathrm{D}$ stack. As one can see from Figure $4, \Delta \mathrm{I}_{\mathrm{ON}}$ is not zero: the packaging resulted in a relative reduction of the saturation currents of n-FETs by approximately -5 to $-7 \%$ in both tiers, while the p-FET sensors react differently on different tiers. The values and the magnitudes of the error bars indicated in Figure 4 correspond respectively to the median values and standard deviations of the shifts of currents calculated based on the measurements of many identical samples (as shown in the last column of Table 1). 
Tier I, nFET

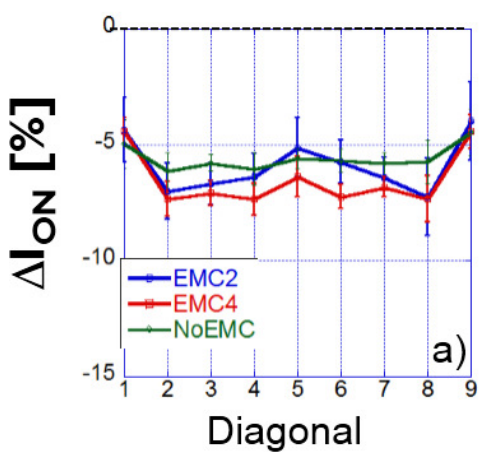

Tier I, pFET

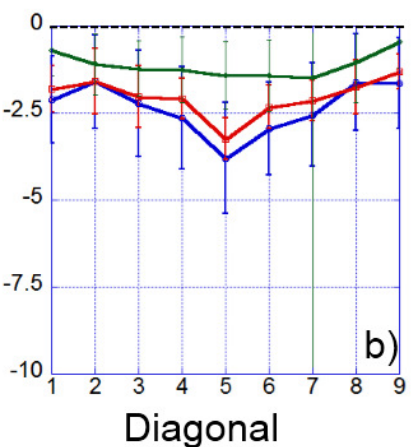

Tier 2, nFET

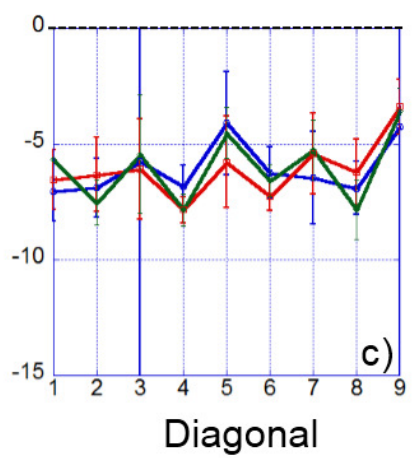

Tier 2, pFET

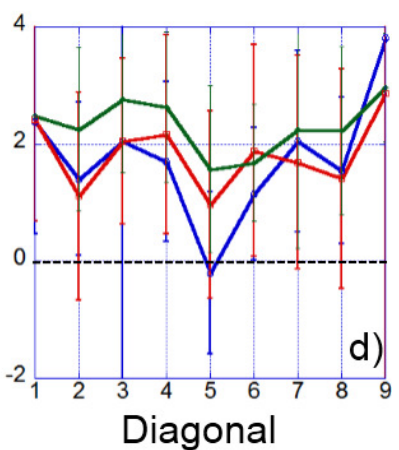

Figure 4. Relative changes of saturation currents $\left(\Delta \mathrm{I}_{\mathrm{ON}}\right)$ of the stress sensors (n- and p-FETs) located along the diagonals of the dies (red arrows in Figure 2) as the result of packaging.

The relationships between mechanical stress $(\sigma)$ induced in $\mathrm{Si}$ and the changes of saturations currents of $\mathrm{n}$ - and p- FETs $\left(\Delta I_{0, n}\right.$ and $\left.\Delta I_{0, p}\right)$ are shown in equation 1 .

$$
\left\{\begin{array}{l}
\Delta I_{0, n}=\pi_{11, n} \sigma_{11}+\pi_{12, n} \sigma_{22}+\pi_{13, n} \sigma_{33} \\
\Delta I_{0, p}=\pi_{11, p} \sigma_{11}+\pi_{12, p} \sigma_{22}+\pi_{13, p} \sigma_{33}
\end{array},\right.
$$

where $\sigma_{11}, \sigma_{22}, \sigma_{33}$ are the three independent normal components of mechanical stress induced in $\mathrm{Si}$ by the packaging process (two in-plane components and one out-ofplane component, respectively).

The coefficients $\pi_{i j, n}, \pi_{i j, p}$ which are used in this equation are the effective piezoresistive coefficients which have been obtained experimentally by calibrating the stress sensors. Coefficients $\pi_{11}$ and $\pi_{12}$ are obtained using the 4-point bending setup [12] which induces a known uniaxial in-plane stress, while the coefficient $\pi_{13, n}$ is calibrated by applying vertical stress to the sensors using nano-indentation technique $[9,13]$. These coefficients are listed in Table 2.

Table 2. Effective piezoresistive coefficients of the n- and p-type FETs.

\begin{tabular}{|c|c|c|c|}
\cline { 2 - 4 } \multicolumn{1}{c|}{} & $\begin{array}{c}\pi_{11} \\
{[\mathbf{p p m} / \mathbf{M P a}]}\end{array}$ & $\begin{array}{c}\pi_{12} \\
{[\mathbf{p p m} / \mathbf{M P a}]}\end{array}$ & $\begin{array}{c}\pi_{13} \\
{[\mathbf{p p m} / \mathbf{M P a}]}\end{array}$ \\
\hline n-FET & 306 & 178 & -560 \\
\hline p-FET & -57 & -204 & $11^{*}$ \\
\hline
\end{tabular}

*The vertical piezoresistive coefficient of p-FET cannot be calibrated experimentally because of the limitation of the current design. Thus, the coefficient shown in the table $\left(\pi_{13}=11\right)$ is taken from [14].

To get an unambiguous solution for the three unknown stress components using two independent measurements of $n$ and p-FETs, a one eight symmetry of the packages is assumed. Therefore, in the diagonal of the die, as illustrated in Figure 2, the in-plane global stress components are identical $\left(\sigma_{11}=\sigma_{22}\right)$. The distributions of in-plane stress induced by the packaging process along the diagonals of the packages in two tiers within the 3D stack calculated using equation 1 and the measurements of stress sensors, are shown in Figure 5.

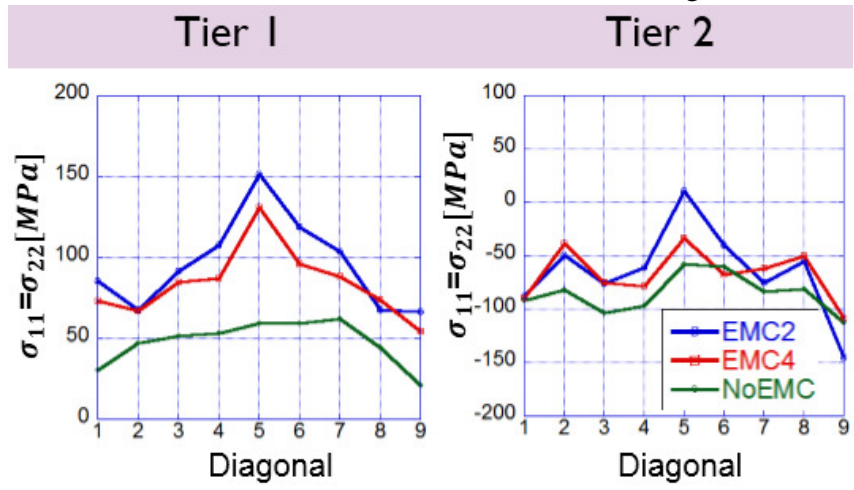

Figure 5. In-plane stress induced in Tier 1 and Tier 2 of the $3 \mathrm{D}$ stack by the packaging process in three different packaging configurations.

It is important to notice that the reported levels of stress are the average numbers calculated based on the measurement of a limited amount of packages. The measurement error bars shown in Figure 4 result in an average inaccuracy of $\pm 50 \mathrm{MPa}$. This inaccuracy is relatively high and is comparable with the observed stress levels. This makes it difficult to compare slight variations in global stress induced by different packaging options but it still allows making general qualitative conclusions about the effects of 3D stacking and packaging on mechanical stress built in Si dies.

It can be seen that the stress levels on Tier 1 and Tier 2 are very different. Thus, in terms of the in-plane stress, packaging adds compressive stress in Tier 2 in the range between -50MPa and $-100 \mathrm{MPa}$. This stress is similar (within the error bars discussed above) in all three packaging configurations including the one with the bare die ("NoEMC" case). This indicates that most of the in-plane stress in Tier 2 is created by the flip-chip die attach while the overmolding process has minor effect on this stress.

As for the stress induced by packaging in Tier 1 (thick die within the 3D stack), the situation is different in comparison with the Tier 2 case. The in-plane stress in Tier 1 is tensile in all three packaging options. The amplitude is below 50MPa in 
the case of the bare packages while addition of the mold compound clearly adds an extra 50MPa-100MPa of stress.

It is worth noting that the above discussed stress is the change of stress due to packaging only. The stress which was build inside the dies before packaging, i.e. during 3D stacking is not considered in Figure 5. The global stress build inside the 3D stack prior to packaging can be calculated by monitoring the deviations of the ION currents of the n-type and p-type stress sensors from their initial (stress-free) values as the result of stacking. These reference currents (non-disturbed by external stress) are measured on many identical sensors $(>100)$ located on different wafers from the same manufacturing lot. The calculated average currents of the sensors and their standard deviations measured at temperature $\mathrm{T}=25^{\circ} \mathrm{C}$ are $\mathrm{I}_{\mathrm{ON}, \text { ref, }, \mathrm{n}-}$ $\mathrm{FET} \cong 16.90 \mu \mathrm{A} \pm 0.25 \mu \mathrm{A}$ and $\mathrm{I}_{\mathrm{ON}, \text { ref, } \mathrm{p}-\mathrm{FET}} \cong 3.39 \mu \mathrm{A} \pm 0.03 \mu \mathrm{A}$ for $\mathrm{n}$ - and p-FETs, respectively. Thus, the calculated global inplane stress induced by the 3D stacking in Si dies is tensile on both tiers (as shown in Figure 6) with an amplitude of $(70 \pm 30) \mathrm{MPa}$.

The resulting in-plane stress shown in Figure 7, which is induced by the complete assembly cycle including 3D stacking and packaging is calculated as an algebraic sum of individual contributions assuming that they are independent and linear.

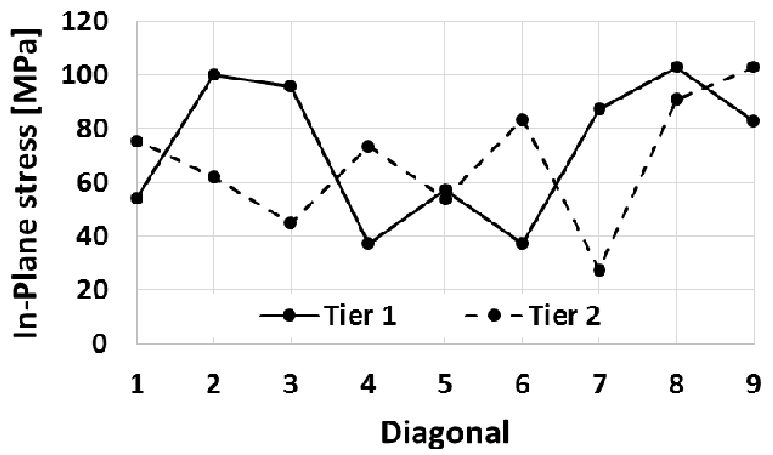

Figure 6. In-plane stress induced in Si dies after the 3D stacking process.

As it can be seen from Figure 7, as the result of stacking and packaging, the total in-plane stress induced in Tier 1 is tensile and about 100MPa in amplitude in the bare-die packages and it increases to (150 to 200)MPa when measuring packages with EMC. On the other hand, the in-plane tensile stress on Tier 2 measured at the stack level (Figure 6) is compensated by the compressive stress induced by the warpage of the laminate substrate (Figure 5).

\section{Effects of packaging on local stress distributions within the 3D stacks}

In [9] it was shown that the 3D stacking assembly process results in significant compressive stress $(\sim-250 \mathrm{MPa})$ locally induced in $\mathrm{Si}$ at the locations below the micro-bumps. This local stress is created due to the combination of high temperature applied to the stack during thermo-compression bonding process and the mismatch in thermo-expansion coefficients of copper and underfill.
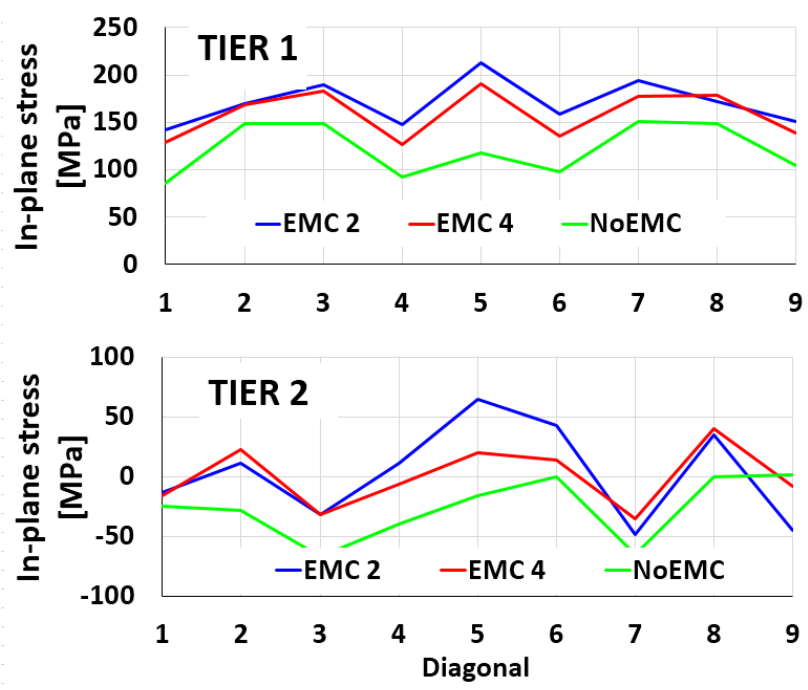

Figure 7. Combined in-plane stress induced in Si dies after the $3 \mathrm{D}$ stacking and packaging.

On the other hand it was shown that the thin $(50 \mu \mathrm{m}$ thick) Tier 2 die stays flat after the assembly due to the fine pitch of the micro-bumps. To observe the influence of different packaging steps (i.e. die attach to the laminate and overmolding) on the local distributions of stress within the 3D stack, the local stress monitors are measured after packaging. These monitors of local stress are the ones reported in [9] and consisting of an array of local n-type stress sensors (green rectangles in Figure 8) located below the micro-bumps on Tier 1 and on top of the stack on Tier 2.

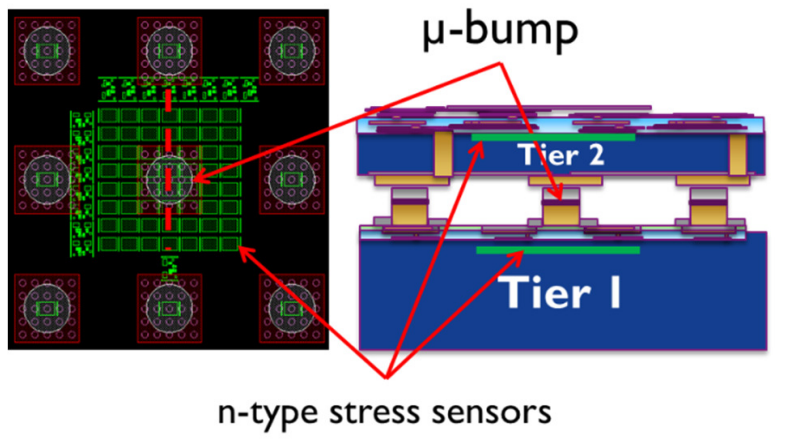

Figure 8. Schematic top view and cross-section of the arrangement of the local stress sensors. Circles indicate locations of micro-bumps and green rectangles/lines show the locations of the stress sensors on two tiers. The red dashed line indicates the positions of the sensors located along the symmetry line of the micro-bump.

The local variations of currents of the stress sensors measured after packaging are compared with those measured on the stack level before packaging. These effects are presented as relative changes of currents of the stress sensors calculated as $\Delta \mathrm{I}=\left(\mathrm{I}_{\mathrm{ON}}-\mathrm{I}_{\mathrm{ON}}, \mathrm{ref}\right) / \mathrm{I}_{\mathrm{ON}}$, ref $100 \%$. 


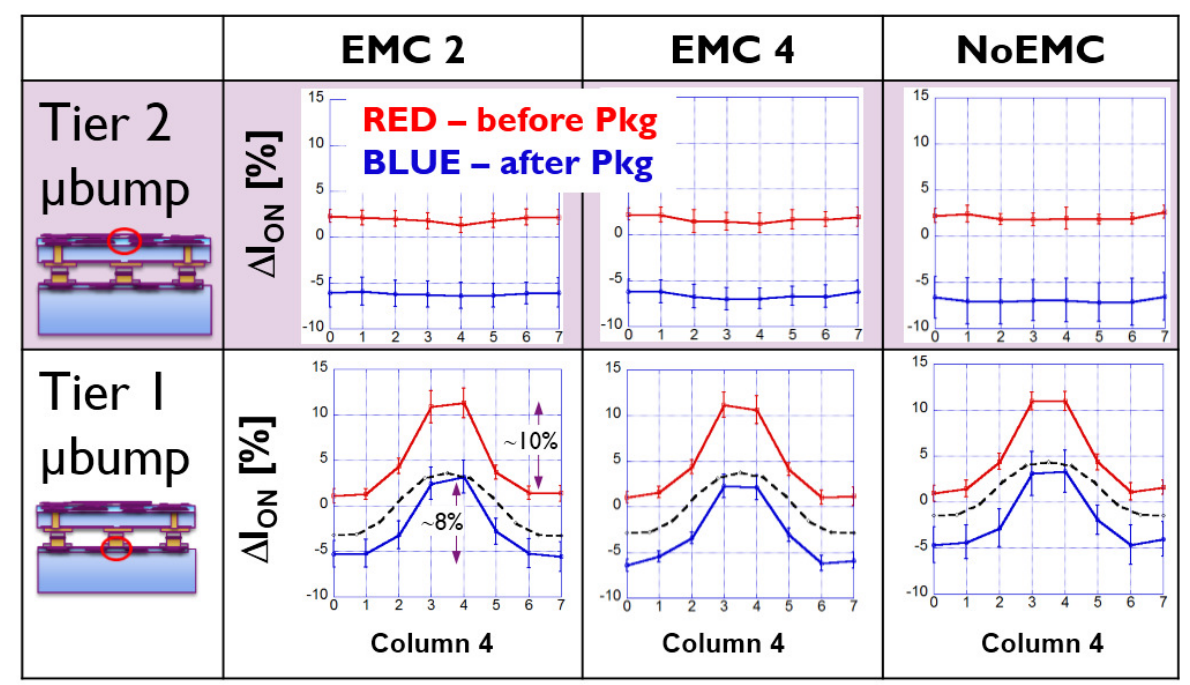

Figure 9. Variations of the ION currents of the local stress sensors along dashed red line from Figure 8 for two different locations within the 3D stacked dies measured before and after packaging for three different packaging options. The locations of the sensors are indicated by the red circles. Dashed black lines are the results of the finite element modeling.

The $\mathrm{I}_{\mathrm{ON}}$, ref are the minimal currents measured within every array of sensors at the stack level. This choice of the reference current allows to compare the effects of packaging on both the shape of the local stress and also the absolute change of stress.

The results of the measurements and of the data analysis are shown in Figure 9 where the variations of the ION currents of the local stress sensors for two different locations within the stacked dies measured before and after packaging for the three different packaging options are compared. It is noticeable that three different packaging options do not significantly change the shape of the stress variations above and below the microbump.

This observation is also confirmed by finite element modeling (FEM) and Raman spectroscopy results. FEM was used to model the components of stress induced in specific locations within the 3D stack. These stress components have been converted into the shifts of currents of the stress sensors using equation 1 and the piezoresistive coefficients from Table 2. The modelled shapes of the shifts of currents are very similar for all three packaging options as it is shown by the dashed lines in Figure 9.

To perform the stress measurements using the microRaman spectroscopy technique [15], the packages were cross sectioned. The planes of the cross sections coincide with the locations of micro-bumps as is shown in Figure 10a. The Raman frequency shifts were measured along the scan line located on the back side of Tier 2 just below the micro-bumps and are very similar for all three packaging options (Figure 10b). This gives an additional confirmation to the above conclusion that the different packaging options investigated in this work have similar effects on the shape and amplitude of stress induced in stacked Si dies.

\section{Discussions on effects of packaging}

From Figure 9 it is seen that the amplitude (peak-to-peak variation) of the current shifts of the sensors located on Tier 1 below the micro-bump reduces from 10 to $11 \%$ measured at the stack level to 6 to $7 \%$ after packaging, indicating a slight relaxation of local stress built during the thermo-compression bonding (TCB). This, in turn, could be an indirect indication of the non-complete formation of intermetallic solder joint within the micro-bump during assembly of the 3D stack so that the following high temperature steps used during flip-chip die attach to the package substrate further affect the microstructure of the micro-bumps.
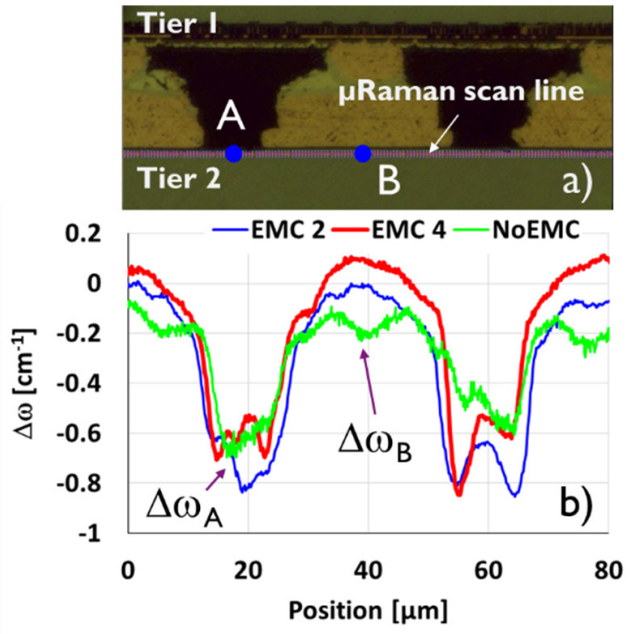

Figure 10. a) Typical view of the local cross section of the package with the indicated micro-Raman spectroscopy scan line and $b$ ) shift of the Raman frequency along the scan line for three packaging options. Points " $A$ " and " $B$ " indicate the positions of maximal and minimal Raman frequency shifts.

It is also noticeable that all graphs reported in Figure 9 for the case of 3D stacks (red curves) are shifted down by 6-8\% as the result of packaging. These shifts are comparable with those reported for the n-type global stress sensors in Figures 4 a,c. From Table 2 it is seen that the n-type sensors have positive inplane piezoresistive coefficients while the vertical coefficient is negative. Thus the negative current shifts can be produced by the in-plane compressive stress induced by the global warpage 
of $\mathrm{Si}$ dies inside the packages or by the high tensile vertical stress between the dies and between the 3D stack and the package substrate. To distinguish between these two effects the warpage of the bare die packages is measured using two different optical techniques. One being the white light interferometry. Another method is based on the Moiré technique and allows to measure the profile of the packages from both sides, molded or non-molded top side of the package and the back side of the package containing the BGA solder balls. The results of the profilometry measurement of the "baredie" package using the Moiré-based technique are shown in Figure 11.
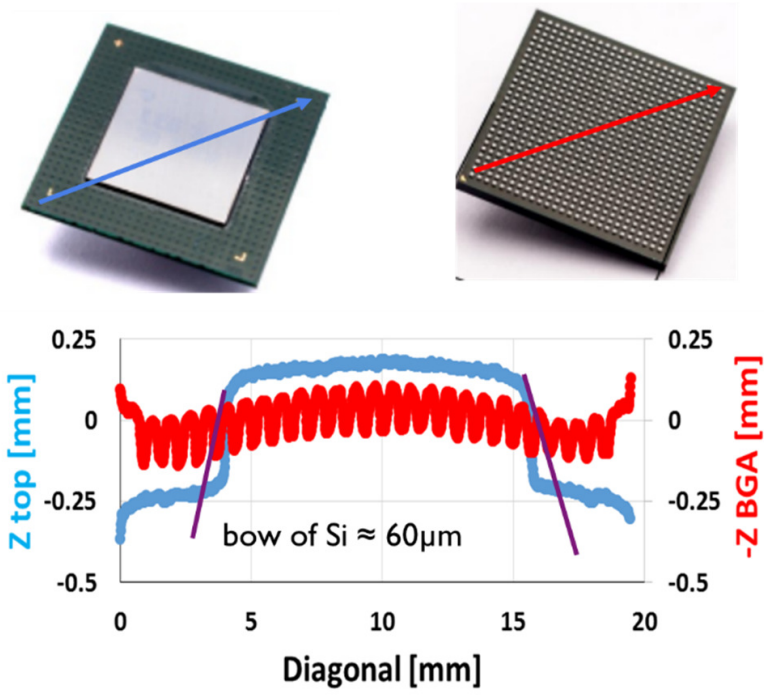

Figure 11. Comparison of two optical profiles taken from both sides of the same non-molded package (NoEMC). To ease the comparison, the profile taken from the back side of the package (side of the BGA solder balls) is inverted.

From this figure it is seen that the warpage of the part of the package within the footprint of the $3 \mathrm{D}$ stack $(8 \mathrm{mmx} 8 \mathrm{~mm})$ is approximately $60 \mu \mathrm{m}$. A similar warpage $(59 \mu \mathrm{m})$ is measured by the white light interferometry from the exposed side of the 3D stack assembled on the laminate substrate. To be able to make a comparison of different packaging options, the warpage of the three samples is measured from the back side of the packages (BGA ball side) and is shown in Figure 12. As it can be seen, the warpage of the bare-die package $(\sim 60 \mu \mathrm{m}$ within the area of the 3D stack), is slightly reduced by the addition of the mold compound.

The convex shape of the packages (if looking from the top side) indicates that the top side of the package containing the 3D stack is likely under tensile in-plane stress (line " $D$ " in Figure 13). This stress will gradually decrease when moving down inside the package and finally change sign to compressive at the level of the laminate substrate. The position of this transition, i.e. the zero-stress line, is very difficult to determine because of the complex combination of different materials within the package.

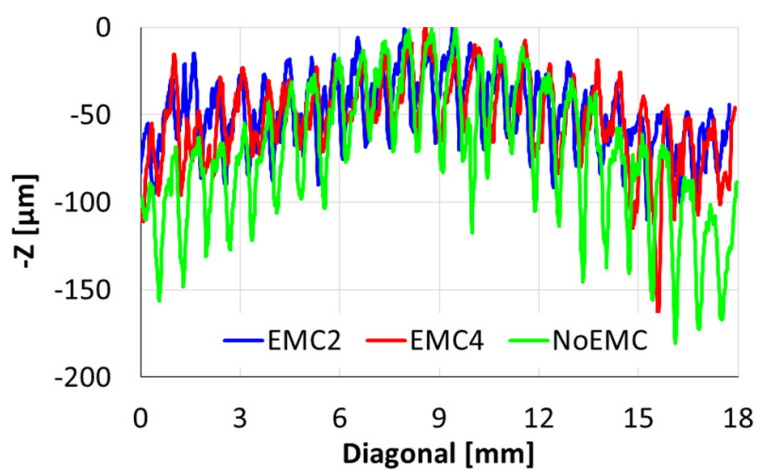

Figure 12. Warpage of three different packages measured from the side of the BGA balls by the Moiré-based technique. All profiles are inverted to ease the comparison.

Thus, the in-plane stress measured by the global stress sensors (in the locations between the micro-bumps) and reported in Figure 7 indicates that the zero-stress line is close to the plane of the stress sensors located on Tier 2 (line " $\mathrm{A}$ " in Figure 13), i.e. the closest one to the package' substrate.

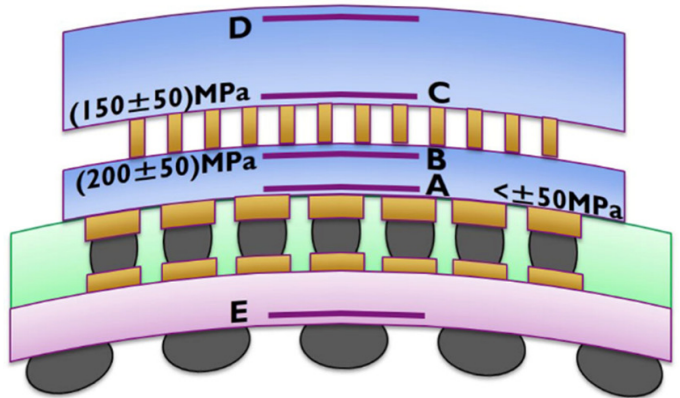

Figure 13. Schematic view on the cross section of the warped bare-die package. Lines "A"-"D" indicate the positions where different techniques are used to estimate the stress: Lines " $A$ " and " $C$ " show the locations of the stress sensors, line " $B$ " indicates the micro-Raman spectroscopy scan line, and the lines " $D$ " and "E" indicate the top and bottom surfaces of the package which are used to measure warpage of the package.

The stress measured by the sensors located on the FEOL side of the Tier 1 and reported in Figure 7 (line " $C$ " in Figure 13) is tensile, as it is expected from the warpage measurement results, and is of $\sim(150 \pm 50) \mathrm{MPa}$ in amplitude. In addition, a detailed analysis of the Raman spectra from Figure 10, as explained in the following, indicates that the back side of the Tier 2 die (line " $B$ " in Figure 13) is under tensile inplane stress with the amplitude of $\sim(200 \pm 50) \mathrm{MPa}$. This conclusion is based on the fact that the amplitude of the Raman frequency shift at the locations between micro-bumps (point "A" in Figure 10) is $\Delta \omega_{A}=-0.6 \mathrm{~cm}^{-1}$. According to the Raman theory applied to [100] oriented Si cross-section surface, this frequency shift corresponds to the following combination of vertical and in-plane components of stress measured in the cross section of the package: 


$$
\Delta \omega_{A(B)}=-2.3 \sigma_{11, A(B)}-2.3 \sigma_{33, A(B)}, \text { Eq. } 2
$$

where $\Delta \omega\left[\mathrm{cm}^{-1}\right]$ is the measured Raman frequency shift and $\sigma_{\mathrm{ij}}[\mathrm{GPa}]$ are the stress components. The coefficients in Eq.2 are calculated based on the parameters reported in $[16,17]$.

Here it is assumed that the stress normal to the cross-section of the package is relaxed and can be neglected. It can also be assumed that the local variation of in-plane stress along the Raman spectroscopy scan line due to the presence of the microbump and the underfill (points "B" and "A" in Figure 10, respectively) is negligible in comparison with vertical stress and with the global in-plane stress induced by the warpage of the package. Thus, one can write:

$$
\sigma_{11, A}=\sigma_{11, B}=\sigma_{11},
$$

where $\sigma_{11}$ is the global part of the in-plane stress induced in Tier 2 due to warpage of the package.

From [9] it is known that the tensile vertical force between micro-bumps induced by the CTE mismatch between copper and underfill is compensated by the compressive reaction force at the center of the micro-bump. That is, the vertical tensile stress between bumps is proportional to the vertical compressive stress at the center of the bump (point " $\mathrm{B}$ " in Figure 10):

$$
\sigma_{33, A} \approx-0.44 \sigma_{33, B},
$$

where the proportionality coefficient is calculated as ratio of areas filled by copper micro-bump $(25 \mu \mathrm{m}$ in diameter $)$ and underfill around the bump ( $40 \mathrm{x} 40 \mu \mathrm{m}$ square).

The system of equations 2, 3 and 4 results in the set of stress components which values are estimated for different locations along the measured scan line. Thus, the warpage-induced global in-plane stress $\sigma_{11}$ is calculated as tensile $\sim(200 \pm 50) \mathrm{MPa}$ whereas the vertical stress between microbumps $\left(\sigma_{33, A}\right)$ is tensile $(80 \pm 50) \mathrm{MPa}$ in amplitude and the reaction compressive stress at the center of the micro-bump $\left(\sigma_{33, B}\right)$ is in the range -150 to -250MPa for all three packaging options.

The combination of the results obtained by to different independent methods (electrical measurement of stress sensors and micro-Raman spectroscopy) indicates that the in-plane stress induced on the back surface of Tier 2 (line "A" in Figure 13) is similar to the in-plane stress induced on the FEOL side of Tier 1 (line "B" in Figure 13) within the accuracy of the measurement techniques. That is, 12 to $15 \mu \mathrm{m}$ thick interface between two Si dies within the 3D stack consisting of microbumps and underfill does not significantly contribute to the gradient of in-plane stress induced by the global warpage of the package. At the same time from [9] it is know that this interface is the major source of vertical stress induced in Si dies.

It should be pointed out that it is very difficult to perform accurate quantitative comparison of the results acquired using different methods because the boundary conditions set by three measurement techniques are very different. Thus, optical warpage measurements are performed on the free-standing samples while the micro-Raman spectroscopy is done on the cross section of the package where it is assumed that one inplane component of stress is completely relaxed. At the same time the electrical measurements of the stress sensors are performed by placing the packages in the socket (as in Figure 3). To insure good and reliable contacts between the BGA solder balls of the package and the contact pads of the socket the package is pressed vertically. Considering significant warpage of packages (around $0.1 \mathrm{~mm}$ as it is reported in Figure 12) it is expected that the vertical force applied to the packages should be high enough to flatten the packages and to make sure that all solder balls are connected to the pads. Such manipulation with warpage of the packages during electrical measurements may further affect the stress evaluated by this technique within the range of $\pm 50 \mathrm{MPa}$.

\section{Summary}

The performed analysis of the experimental data obtained in this work using different techniques (electrical, optical) has shown that:

1. The influence of all packaging steps (including flipchip die attach to the laminate substrate and application of the electronic mold compound) on the shape of the local distributions of mechanical stress around the micro-bump induced after 3D stacking is, in general, non-significant, while the vertical stress in Tier 1 under the micro-bump is slightly reduced as the result of packaging (as it is shown in Figure 9).

2. The absolute levels of local stress are significantly affected by the packaging. By comparing the packaging option with bare die (NoEMC) with other packaging options (Figure 9 and Figure 10) it is noticeable that the main contribution to the change in stress is coming from the flip-chip die attach to the laminate substrate while the following overmolding process does not significantly affect the stress levels.

3. Analysis of the global stress sensors (Figure 7), the results of the warpage measurements (Figure 11) and the micro-Raman spectroscopy confirms the previous conclusion that the packaging process affects stress in both tiers within the 3D stack where the processes of flip-chip die attach to the substrate plays the dominant role.

\section{Conclusions}

Flip-chip BGA packages containing the 3D-stacking enabled test chips were used to investigate the effects of different assembling steps such as flip-chip die-attach to the laminate substrate, underfilling and overmolding on the redistribution of mechanical stresses in 3D-ICs. The observed effects are supported by the finite element modeling, optical warpage measurements and by the micro-Raman spectroscopy.

\section{Acknowledgments}

The authors would like to thank core partners of the imec Industrial Affiliation Program (IIAP) on 3D Integration Technology.

\section{References}

1. E. Beyne, The rise of the 3rd dimension for system intergration. Interconnect Technol. Conf. 2006, 1-5 (2006).

2. G. Van Der Plas et al., Design issues and considerations for low-cost 3-D TSV IC technology. Solid-State Circuits, 46 , 293-307

(2011). 
3. G. Vakanas et al. Metallurgies evaluation ( $\mathrm{Sn}$ vs. $\mathrm{SnCu} 0.7 \%$ vs. SnAg) for 3D bumping and stacking. Presented at TMS 2014, $143^{\text {rd }}$ Annual meeting and Exhibition.

4. J. Derakhshandeh et al., Reflow process optimization for micro-bumps applications in 3D technology, Electron. Syst , pp. 2-6, 2014.

5. L. Lin. et al. Reliability characterization of Chip-onWafer-on-Substrate (CoWoS) 3D IC integration technology. 2013 IEEE 63rd Electron. Components Technol. Conf. 366-371 (2013).

6. V.O. Cherman et al. Impact of through silicon vias on front-end-of-line performance after thermal cycling and thermal storage. IEEE Int. Reliab. Phys. Symp. 2B.3.12B.3.5 (2012).

7. S. Cho et al. Impact of TSV proximity on 45nm CMOS devices in wafer level. 2011 IEEE Int. Interconnect Technol. Conf. 1-3 (2011).

8. J. Auersperg et al., Nonlinear copper behavior of TSV and the cracking risks during BEoL-built-up for 3D-ICintegration, ... Multi-Physics ..., pp. 1-6, 2012.

9. V. Cherman et al., 3D stacking induced mechanical stress effects, in (ECTC), 2014 IEEE ..., 2014, pp. 309-315.

10. J. De Vos et al., Comparative study of 3D stacked IC and 3D interposer integration: processing and assembly challenges, 3D Syst. Integr. Conf., 2014.

11. H. Oprins et al. Experimental thermal characterization and thermal model validation of 3D packages using a programmable thermal test chip, in (ECTC), 2015 to be published.

12. A. Ivankovic et al., "FET arrays as CPI sensors for 3D stacking and packaging characterization," 2012 IEEE Int. Reliab. Phys. Symp., pp. 2E.3.1-2E.3.9, Apr. 2012.

13. K. Vanstreels et al., Advanced experimental back-end-ofline (BEOL) stability test: Measurements and simulations, Microelectron. Eng., Oct. 2014.

14. J. C. Suhling and R. C. Jaeger, "Silicon piezoresistive stress sensors and their application in electronic packaging," IEEE Sens. J., vol. 1, no. 1, pp. 14-30, Jun. 2001.

15. I. De Wolf. Micro-Raman spectroscopy to study local mechanical stress in silicon integrated circuits. Semicond. Sci. Technol. 11, 139-154 (1996).

16. E. Anastassakis et al., Piezo-Raman measurements and anharmonic parameters in silicon and diamond, vol. 41, no. 11, pp. 7529-7535, 1990.

17. W. A. Brantley, Calculated elastic constants for stress problems associated with semiconductor devices, vol. 44, no. 1, pp. 1972-1973, 1973. 